\title{
Phosphatidylinositol 3-kinase p110a mediates phosphorylation of AMP-Activated Protein Kinase in myoblasts
}

\author{
Ronald W. Matheny Jr. ${ }^{\text {a\#, }}$, Alyssa V. Geddis ${ }^{\text {a }}$, Mary N. Abdalla ${ }^{\mathrm{a}, 1}$, and Luis A. Leandry ${ }^{\mathrm{a}}$
}

Running title: PI3K p110 regulates AMPK phosphorylation

${ }^{a}$ Military Performance Division, US Army Research Institute of Environmental Medicine, 15 Kansas Street, Building 42, Natick, MA, 01760 USA.

${ }^{1}$ Present address: University of Massachusetts Medical School, Department of Molecular Cell and Cancer Biology, 364 Plantation St., Worcester, MA 01605

\# To whom correspondence should be addressed: Ronald W. Matheny Jr., Military Performance Division, US Army Research Institute of Environmental Medicine, 10 General Greene Ave, Building 42, Natick, MA, 01760, USA, Tel.: (508) 233-5420; Fax: (508) 233-4195; email: ronald.w.matheny.civ@mail.mil 


\begin{abstract}
AMP-activated protein kinase (AMPK) is a serine/threonine kinase that functions as a sensor of intracellular energy. Activation of AMPK is associated with increased phosphorylation of the $\alpha$ subunit at threonine 172 (T172) and decreased phosphorylation at serine 485 in AMPK $\alpha 1$ and serine 491 in AMPKa2 (S485/491). One potential mediator of AMPK phosphorylation is phosphatidylinositol 3-kinase (PI3K); however, the mechanism and the identities of the specific PI3K isoforms that regulate AMPK activation are not known. To determine whether PI3K p110 $\alpha$ regulated AMPK activation in muscle cells, C2C12 myoblasts were subjected to pharmacological inhibition of $\mathrm{p} 110 \alpha$, siRNA directed against $\mathrm{p} 110 \alpha$, or overexpression of constitutively-active or dominant negative p110 $\alpha$. Chemical inhibition, siRNA, and expression of dominant-negative p110 $\alpha$ were all associated with increased AMPK T172 phosphorylation, whereas expression of constitutively-active p110 $\alpha$ reduced T172 phosphorylation. Conversely, pharmacological inhibition of p110 $\alpha$ reduced AMPK S485/491 phosphorylation, while constitutively-active p110 $\alpha$ increased AMPK S485/491 phosphorylation. This p110 $\alpha$-mediated increase in AMPK S485/491 phosphorylation was eliminated in the presence of the Akt inhibitor MK2206, suggesting that p110 $\alpha$-mediated phosphorylation of AMPK $\alpha$ at S485/491 is Aktdependent. In response to oligomycin or serum-starvation, AMPK T172 phosphorylation was elevated in p110 $\alpha$-deficient myoblasts compared to control myoblasts. Overall, our findings identify PI3K p110 $\alpha$ as a mediator of AMPK phosphorylation in myoblasts.
\end{abstract}

Key words: Phosphatidylinositol 3-kinase (PI3K), AMP-Activated Protein Kinase (AMPK), p110 $\alpha$, Akt, myoblast 


\section{Abbreviations:}

AMPK: Adenosine monophosphate-Activated Protein Kinase

PI3K: phosphatidylinositol 3-kinase

Akt: homologue to the oncogene in transforming retrovirus AKT-8

siRNA: small-interfering RNA

PRAS40: proline-rich Akt substrate of $40 \mathrm{kDa}$

\section{Introduction}

AMP-activated protein kinase (AMPK) is a heterotrimeric serine/threonine kinase that acts as a sensor and regulator of cellular energy status. In skeletal muscle, AMPK regulates multiple metabolic and energetic processes including glucose uptake, fatty acid oxidation, and mitochondrial biogenesis [1]. AMPK is activated in response to low cellular energy (high AMP:ATP ratio) and physical exercise $[2,3,4]$ and this activation is generally associated with phosphorylation of the $\alpha$-subunit at threonine 172 (T172). AMPK inhibition on the other hand, is associated with phosphorylation at serine 485 in AMPK $\alpha 1$ and serine 491 in AMPK $\alpha 2$ (S485/491) in several cell types, including skeletal muscle [5,6,7,8,9]. A number of upstream regulators of AMPK have been identified that either positively or negatively modify AMPK activity [10], and the coordination of these inputs maintains metabolic and energetic homeostasis. 
It has recently been reported that insulin and IGF-I mediate the phosphorylation of AMPK S485/491 through a wortmannin-sensitive mechanism in skeletal muscle, suggesting the involvement of phosphoinositide 3-kinase (PI3K) [9]. PI3K is a heterodimeric protein and lipid kinase expressed in a number of tissues, including skeletal muscle. Class IA PI3K enzymes are composed of an $85 \mathrm{kDa}$ regulatory subunit (p85) and a $110 \mathrm{kDa}$ catalytic subunit (p110) and are recruited to the cell membrane following activation of receptor tyrosine kinases (RTK) and G protein-coupled receptors (GPCRs). In skeletal muscle, three Class IA PI3K catalytic isoforms are expressed (p110 $\alpha, \mathrm{p} 110 \beta$, and $\mathrm{p} 110 \delta)[11,12]$. While $\mathrm{p} 110 \alpha$ is the primary $\mathrm{p} 110$ isoform regulating cellular responses to RTK agonists such as insulin and IGF-I [13,14], p110 3 is the primary p110 isoform regulating cellular responses to GPCR agonists such as lysophosphatidic acid $[15,16]$. However, the role of PI3K in regulating AMPK activation, and the identity of the catalytic subunits involved, are not known.

Given the importance of PI3K p110 $\alpha$ in regulating insulin/IGF signal transduction in skeletal muscle, and given that AMPK activation is sensitive to PI3K pharmacological inhibition, we performed a series of experiments in cultured myoblasts to determine whether p110 $\alpha$ regulated AMPK phosphorylation. Our findings demonstrate that PI3K p110 $\alpha$ mediates AMPK phosphorylation under a variety of experimental conditions.

\section{Materials and Methods}

Materials and Reagents. C2C12 cells and DMSO were purchased from American Type Culture Collection (ATCC, Manassas, VA). Dulbecco's modified Eagle's mediun (DMEM), fetal bovine serum (FBS), penicillin/streptomycin, trypsin, Lipofectamine 2000, and Opti-mem 
medium were purchased from Life Technologies (Carlsbad, CA). MK2206 was from Selleck Chemicals (Houston, TX). PI3-K alpha inhibitor 2 (cat \# B-0304, corresponds to Figure 15e in reference [17] and referred to as p110ai in this manuscript) was purchased from Echelon Biosciences (Salt Lake City, UT). Antibodies for p-AMPK $\alpha$ Thr 172 (\#2535), p-AMPKa Ser 485/491 (\#4185), total AMPKa (\#2603), p-Akt Ser 473 (\#4060), total (pan) Akt (\#4685), pPRAS40 Thr 246 (\#2997), total PRAS40 (\#2691), p110a (\#4249), p-S6 Ser 240/244 (\#4838), total S6 (\#2217), and oligomycin (\#9996) were purchased from Cell Signaling Technologies (Danvers, MA). HRP-linked secondary antibodies were obtained from Santa Cruz Biotechnology (Santa Cruz, CA). All other chemicals and reagents were purchased from Sigma (St. Louis, MO).

Cell culture and pharmacological inhibitors. $\mathrm{C} 2 \mathrm{C} 12$ were grown and passaged in highglucose DMEM containing $10 \% \mathrm{FBS}$ and $1 \%$ antibiotics at $37^{\circ} \mathrm{C}$ in $5 \% \mathrm{CO}_{2}$. All experiments were performed within six passages of receipt from the vendor (ATCC). Following reconstitution, cells were expanded for a minimum of five days prior to any experimental manipulations. Chemical inhibitors p110ai and MK2206 were dissolved in DMSO and diluted in culture medium such that cells were exposed to a final DMSO concentration of $0.02 \%$. Control cells received an equivalent concentration of DMSO.

Small-interfering RNA (siRNA). Non-targeting control siRNA ( \#4390483) and siRNA directed against p110 (\#s71604) were purchased from Life Technologies. Cells were reversetransfected in DMEM containing 10\% FBS without antibiotics at a density of $0.95 \times 10^{4}$ cells $/ \mathrm{cm}^{2}$ using $0.2 \%$ ( $\mathrm{vol} / \mathrm{vol}$ ) Lipofectamine 2000 and a $5 \mathrm{nM}$ final concentration for siRNAs. Twenty four hours following seeding, medium was replaced with DMEM $+10 \%$ FBS with antibiotics for another 24 hours whereupon cells were used for experiments. 
Baculoviruses and transductions. For delivery and expression of genes in cultured cells, we utilized non-replicating baculoviruses that had been modified by insertion of a mammalian expression cassette (Life Technologies). Open reading frame (ORF) clone encoding human p110 $\alpha$ (PIK3CA; \#IOH3620) in the pENTR221 vector was purchased from Life Technologies. The PIK3CA-E545K and PIK3CA-K802R point mutations were introduced by standard PCRbased site-directed mutagenesis. Sequence-verified clones were subcloned into a pDEST8CMV vector as described previously [18]. These expression constructs were transformed into DH10BAC bacteria cells to generate and isolate bacmid DNA. This PCR-qualified bacmid DNA was transfected into SF9 insect cells for BacMam virus production according to Bac-to-Bac ${ }^{\circledR}$ Baculovirus Expression Systems manual [19,20] (Life Technologies). Viral transductions were performed at the time of seeding using 10\% (v/v) virus in antibiotic-free DMEM + 10\% FBS containing 0.02\% enhancer solution (\#PV5835, Life Technologies). Medium was replaced 16hours following transduction and cells were maintained for an additional 24-hours before experiments.

Protein extraction and immunoblotting. Extraction of protein from cells was performed as described [21], and protein concentrations were determined by the method of Bradford [22]. Western immunoblotting was performed exactly as described previously [23], and densiometric analysis was performed on scanned images converted to 8-bit TIFF using NIH Image J 1.60 (http://rsbweb.nih.gov/ij/).

Statistics. Data are presented as mean \pm S.E.M. Statistics were performed using Student's ttest, one-way ANOVA with Dunnett test performed post hoc, or two-way ANOVA with Bonferroni test performed post hoc as indicated in figure legends. A P-value $<0.05$ was considered significant. 


\section{Results}

PI3K p110a regulates AMPK phosphorylation at Thr 172 and Ser 485/491

To determine whether PI3K p110 $\alpha$ regulated AMPK activation in muscle cells, we first treated $\mathrm{C} 2 \mathrm{C} 12$ myoblasts with increasing concentrations of a pharmacological inhibitor of $\mathrm{p} 110 \alpha$ ("p110ai”) [17]. Following 4 hours of treatment, we observed a dose-dependent increase in AMPK phosphorylation at T172, and a decrease in phosphorylation of AMPK at S485/481 (Figure 1A). Decreased phosphorylation of Akt (S473) and S6 (S240/244) verified the efficacy of $\mathrm{p} 110 \alpha$ inhibition. We next tested whether siRNA-mediated reduction of $\mathrm{p} 110 \alpha$ produced similar results. In p110 $\alpha$-deficient myoblasts, phosphorylation of AMPK was increased by 3.6fold as compared to myoblasts treated with control siRNA, whereas no differences in in phosphorylation of AMPK at S485/491 were observed (Figure 1B and 1C). We also observed an $\sim 44 \%$ reduction in phosphorylated Akt at S473 in p110 $\alpha$-deficient myoblasts compared to control myoblasts. Finally, we asked whether forced expression of a constitutively-active form of p110 ("p110 $\alpha$-E545K") produced the opposite effect; that is, whether p110 $\alpha$ inhibited AMPK phosphorylation at T172. We found that, indeed, phosphorylation of AMPK was reduced by $\sim 54 \%$ in cells expressing constitutively-active p110 $\alpha$. Additionally, myoblasts expressing p110 $\alpha$-E545K showed significantly increased ( 2.1-fold) phosphorylation of AMPK at S485/491 (Figure 1D and 1E). In myoblasts expressing a kinase-inactive form of $\mathrm{p} 110 \alpha$ (" $\mathrm{p} 110 \alpha-$ K802R"), phosphorylation of AMPK T172 was increased $\sim 50 \%$, although no change in phosphorylation of AMPK S485/491 was observed (Figure 1C and 1D). Collectively, these data reveal that $\mathrm{p} 110 \alpha$ negatively regulates phosphorylation of AMPK at T172, and that under 
conditions of $\mathrm{p} 110 \alpha$ hyper-activation, $\mathrm{p} 110 \alpha$ positively regulates phosphorylation of AMPK at S485/491.

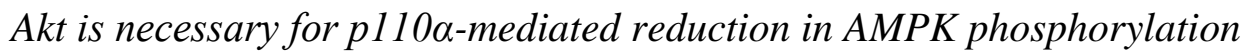

The serine-threonine kinase Akt lies downstream of p110 $\alpha$ and regulates multiple cellular functions including growth factor signaling, survival, and metabolism [24]. With regard to AMPK, Akt has been shown to negatively regulate AMPK activation in cardiac myocytes [8], MCF-7 breast cancer cells [25], and vascular smooth muscle cells [7]. Akt has also been shown to regulate phosphorylation of AMPK $\alpha$ at S485/491 both in vitro [6] and in vivo [26]. Given these reports, we performed a series of experiments designed to determine whether Akt mediated AMPK phosphorylation in myoblasts.

We first established whether pharmacological inhibition of Akt affected phosphorylation of AMPK by using the Akt inhibitor MK2206 [27]. Phosphorylation of AMPK S485/491 was decreased in a dose-dependent fashion in the presence of MK2206; however, MK2206 had no effect on phosphorylation of AMPK T172 (Figure 2A). We next asked whether inhibition of Akt altered p110 $\alpha$-mediated phosphorylation of AMPK. Control myoblasts or myoblasts expressing p110 $\alpha$-E545K were treated with inhibitor vehicle (DMSO) or 125 nM MK2206, a concentration sufficient to inhibit phosphorylation Akt and the Akt substrate PRAS40 (FIG 2A). Expression of p110 $\alpha$-E545K decreased AMPK phosphorylation at T172 and increased phosphorylation of AMPK at S485/491, whereas treatment with MK2206 alone had no statistical effect on AMPK phosphorylation at either residue (Figure 2B and 2C). In the presence of MK2206 however, the p110 $\alpha$-E545K-induced decrease in p-AMPK T172, and the p110 $\alpha$-E545K-induced increase in p- 
AMPK 485/491, were restored to levels comparable to control cells. These data indicate that Akt mediates p110 $\alpha$-induced AMPK phosphorylation in C2C12 myoblasts.

p110a deficiency modifies AMPK responses to cellular stress

Given our observations that $\mathrm{p} 110 \alpha$ regulated AMPK under basal conditions, we next asked whether $\mathrm{p} 110 \alpha$ also regulated AMPK under conditions of cellular stress. We first treated p110 $\alpha$-sufficient or p110 $\alpha$-deficient myoblasts with oligomycin, a known activator of AMPK [28], and analyzed phosphorylation of AMPK. Administration of oligomycin increased phosphorylation of AMPK at T172 in myoblasts treated with control siRNA by $~ 2.1$-fold. In p110 $\alpha$-deficient myoblasts, oligomycin promoted AMPK T172 phosphorylation beyond levels observed in p110 $\alpha$-deficient cells treated with vehicle (Figure 3A and 3B). We also observed an 2.2-fold increase in AMPK $\alpha$ S485/491 phosphorylation in response to oligomycin treatment in siRNA control myoblasts, although this increase was not evident in p110 $\alpha$-deficient myoblasts (Figure 3A and 3B). Oligomycin increased Akt phosphorylation by $~ 2.5$-fold in control cells, but this increase was eliminated in p110 $\alpha$-deficient myoblasts. These data reveal an additive effect of oligomycin and p110 $\alpha$ deficiency on phosphorylation of AMPK at T172 and suggest that $\mathrm{p} 110 \alpha$ mediates oligomycin-induced phosphorylation of AMPK at S485/491.

Previous research has shown that incubation of $\mathrm{C} 2 \mathrm{C} 12$ cells in serum-free media resulted in increased AMPK T172 phosphorylation [29]. To determine whether p110 $\alpha$ mediated AMPK phosphorylation during serum-starvation, control myoblasts or myoblasts deficient in $\mathrm{p} 110 \alpha$ were exposed to serum-free media for periods up to two hours. Western blotting revealed that phosphorylation of AMPK at T172 was increased by serum starvation in a time-dependent 
manner; furthermore, AMPK T172 phosphorylation was significantly increased in p110 $\alpha$ deficient cells relative to control myoblasts at all time points examined (Figure 3C and 3D). Exposure to serum-free media decreased AMPK S485/491 phosphorylation by $68-74 \%$ at all times in both control and p110 $\alpha$-deficient myoblasts, while expression of phosphorylated Akt at S473 was completely eliminated in serum-free media (Figure 3C and 3D). These data demonstrate that during serum-starvation, loss of $\mathrm{p} 110 \alpha$ increases phosphorylation of AMPK at T172 to levels beyond those induced by serum-free media alone.

\section{Discussion}

In this study, we found that PI3K p110 $\alpha$ mediated AMPK phosphorylation in C2C12 myoblasts. In myoblasts treated with a pharmacological inhibitor of p110 $\alpha$, treated with siRNA directed against p110 $\alpha$, or over-expressing kinase-inactive p110 $\alpha$, levels of phosphorylated AMPK at T172 were markedly increased (Figure 1 and Figure 3), whereas in cells expressing constitutively-active p110 , levels of phosphorylated AMPK at T172 were significantly decreased. These findings provide convincing evidence that $\mathrm{p} 110 \alpha$ negatively regulates AMPK T172 phosphorylation; however, the mechanism through which this occurs is less clear. While it has previously been shown that phosphorylation of AMPK at S485/491 inhibits AMPK T172 phosphorylation [6,7], we found that p110 $\alpha$-deficient myoblasts (or myoblasts expressing p110 $\alpha$ K802R) possessed increased AMPK phosphorylation at T172 without a corresponding change in

phosphorylation at S485/491 (Figure 1). Thus, it appears that phosphorylation of AMPK at T172 may be regulated through a mechanism other than AMPK S485/491 phosphorylation, at least under these conditions. In support of this, it was notable that in both p110 $\alpha$-deficient myoblasts 
and in myoblasts expressing $\mathrm{p} 110 \alpha-\mathrm{K} 802 \mathrm{R}$, phosphorylation of Akt was reduced, but not completely eliminated. Since Akt is a downstream mediator of p110 $\alpha$ action, and since Akt has been shown to phosphorylate AMPK at S485/491 [30], it would be expected that a reduction in Akt activation would translate to reduced phosphorylation of AMPK at S485/491. It is possible however, that the residual, active Akt may have been sufficient to maintain levels of phosphorylated AMPK S485/491 in p110 $\alpha$-deficient and p110 $\alpha$-K802R expressing myoblasts at levels comparable to control myoblasts. Another possibility is that molecules other than Akt act to sustain AMPK S485/491 phosphorylation in p110 $\alpha$-deficient myoblasts and in myoblasts expressing $\mathrm{p} 110 \alpha-\mathrm{K} 802 \mathrm{R}$. For example, in non-muscle cells, protein kinase A [30] and p70S6 kinase [31] have both been shown to regulate AMPK phosphorylation at S485/491 under various conditions. Finally, it is possible that loss of p110 $\alpha$ activity results in inhibition of an unidentified AMPK phosphatase. In any case, it is clear that the increased phosphorylation of AMPK at T172 observed in p110 $\alpha$-deficient myoblasts, or in myoblasts expressing p110 $\alpha$ K802R, was not associated with decreased AMPK S485/491 phosphorylation under all experimental conditions (Figure 4).

The role of p110 $\alpha$ in regulating phosphorylation of AMPK at S485/491 however, appears more straightforward. Indeed, myoblasts expressing p110 $\alpha$-E545K displayed robust induction of AMPK S485/491 phosphorylation, an effect that was sensitive to pharmacological inhibition of Akt (Figure 2). These data thus support the existence of a p110 //Akt/AMPK pathway in C2C12 myoblasts. In contrast to p110 $\alpha$-deficient myoblasts (which showed induction of AMPK phosphorylation at T172 but no change in AMPK phosphorylation at S485/491 compared to control cells (Figure 1)), myoblasts expressing p110 $\alpha$-E545K exhibited reduced phosphorylation of AMPK at T172. This finding is in agreement with previous research reporting that AMPK 
S485/491 inhibits phosphorylation of AMPK at T172 in response to insulin or IGF-I [6,7,9], suggesting that this phenomenon also occurs in $\mathrm{C} 2 \mathrm{C} 12$ myoblasts expressing constitutivelyactive $\mathrm{p} 110 \alpha$ (Figure 4).

Interestingly, both AMPK T172 and S485/491 were elevated in response to oligomycin alone, indicating that the inverse relationship between phosphorylation of AMPK T172 and S485/491 observed in response to p110 $\alpha$ expression (Figure 1D an 1E) was not manifest in response to oligomycin. The simplest explanation for this finding is that oligomycin and $\mathrm{p} 110 \alpha$ act through separate mechanisms to mediate phosphorylation of AMPK at T172. We also found that phosphorylation of Akt S473 was elevated in response to oligomycin alone. While this observation supports that Akt acts to phosphorylate AMPK at S485/491, it does not support that AMPK T172 necessarily decreases in response to increased AMPK at S485/491 phosphorylation.

Phosphorylation of AMPK at S485/491 and phosphorylation of Akt at S473 were significantly reduced in serum-free media. While these latter observations are consistent with the idea that low levels of phosphorylated AMPK at 485/491 are permissive for high levels of phosphorylated AMPK at T172 [7], the extent of reduction in AMPK S485/491 phosphorylation did not correlate with the extent of induction of AMPK T172 phosphorylation. Indeed, phosphorylation of AMPK at T172 was increased to various degrees in response to serum-free media and p110 $\alpha$ deficiency, whereas phosphorylation of AMPK at S485/491 was decreased by a relatively constant $\sim 70 \%$ in both p $110 \alpha$-sufficient and p110 $\alpha$-deficient cells throughout the entire time in serum-free media (Figure 3C and 3D). This finding suggests that reductions in phosphorylation of AMPK at S485/491 do not proportionally translate to increased AMPK T172 phosphorylation under these conditions. 
In summary, we identified PI3K p110 $\alpha$ as a principal regulator of AMPK phosphorylation in myoblasts. Actions of p110 $\alpha$ on AMPK were found to be sensitive to pharmacological inhibition of Akt, suggesting a key role for Akt in p110 $\alpha$-AMPK signal transduction. In p110 $\alpha$-deficient myoblasts, phosphorylation of AMPK at T172 was further altered under conditions of cellular stress, demonstrating that $\mathrm{p} 110 \alpha$ acts as a negative regulator of T172 phosphorylation under a variety of experimental conditions. The precise role of p110 $\alpha$ in mediating phosphorylation of AMPK at S485/491, as well as a potential relationship between phosphorylation of T172 and S485/491, remains to be established in this model system.

\section{Acknowledgements}

A.V.G. and M.N.A. were supported by appointments to the Postgraduate Research Participation Program at the U.S. Army Research Institute of Environmental Medicine administered by the Oak Ridge Institute for Science and Education through interagency agreement between the U.S. Department of Energy and U.S. Army Medical Research and Materiel Command.

The views, opinions, and/or findings in this report are those of the authors, and should not be construed as an official Department of the Army position, policy, or decision, unless so designated by other official documentation. 


\section{References}

[1] K.A. Coughlan, R.J. Valentine, N.B. Ruderman, A.K. Saha, AMPK activation: a therapeutic target for type 2 diabetes?, Diabetes Metab Syndr Obes 7 (2014) 241-253.

[2] W.W. Winder, D.G. Hardie, Inactivation of acetyl-CoA carboxylase and activation of AMPactivated protein kinase in muscle during exercise, Am J Physiol 270 (1996) E299-304.

[3] J.F. Wojtaszewski, P. Nielsen, B.F. Hansen, E.A. Richter, B. Kiens, Isoform-specific and exercise intensity-dependent activation of 5'-AMP-activated protein kinase in human skeletal muscle, J Physiol 528 Pt 1 (2000) 221-226.

[4] Z.P. Chen, T.J. Stephens, S. Murthy, B.J. Canny, M. Hargreaves, L.A. Witters, B.E. Kemp, G.K. McConell, Effect of exercise intensity on skeletal muscle AMPK signaling in humans, Diabetes 52 (2003) 2205-2212.

[5] C. Berggreen, A. Gormand, B. Omar, E. Degerman, O. Goransson, Protein kinase B activity is required for the effects of insulin on lipid metabolism in adipocytes, Am J Physiol Endocrinol Metab 296 (2009) E635-646.

[6] S. Horman, D. Vertommen, R. Heath, D. Neumann, V. Mouton, A. Woods, U. Schlattner, T. Wallimann, D. Carling, L. Hue, M.H. Rider, Insulin antagonizes ischemia-induced Thr172 phosphorylation of AMP-activated protein kinase alpha-subunits in heart via hierarchical phosphorylation of Ser485/491, J Biol Chem 281 (2006) 5335-5340.

[7] J. Ning, G. Xi, D.R. Clemmons, Suppression of AMPK activation via S485 phosphorylation by IGF-I during hyperglycemia is mediated by AKT activation in vascular smooth muscle cells, Endocrinology 152 (2011) 3143-3154. 
[8] C.L. Soltys, S. Kovacic, J.R. Dyck, Activation of cardiac AMP-activated protein kinase by LKB1 expression or chemical hypoxia is blunted by increased Akt activity, Am J Physiol Heart Circ Physiol 290 (2006) H2472-2479.

[9] R.J. Valentine, K.A. Coughlan, N.B. Ruderman, A.K. Saha, Insulin inhibits AMPK activity and phosphorylates AMPK Ser(4)(8)(5)/(4)(9)(1) through Akt in hepatocytes, myotubes and incubated rat skeletal muscle, Arch Biochem Biophys 562 (2014) 62-69.

[10] D.G. Hardie, The AMP-activated protein kinase pathway--new players upstream and downstream, J Cell Sci 117 (2004) 5479-5487.

[11] B. Geering, P.R. Cutillas, G. Nock, S.I. Gharbi, B. Vanhaesebroeck, Class IA phosphoinositide 3-kinases are obligate p85-p110 heterodimers, Proc Natl Acad Sci U S A 104 (2007) 7809-7814.

[12] R.W. Matheny, Jr., M.L. Adamo, PI3K p110 alpha and p110 beta have differential effects on Akt activation and protection against oxidative stress-induced apoptosis in myoblasts, Cell Death Differ 17 (2010) 677-688.

[13] L.C. Foukas, M. Claret, W. Pearce, K. Okkenhaug, S. Meek, E. Peskett, S. Sancho, A.J. Smith, D.J. Withers, B. Vanhaesebroeck, Critical role for the p110alpha phosphoinositide-3-OH kinase in growth and metabolic regulation, Nature 441 (2006) 366-370.

[14] Z.A. Knight, B. Gonzalez, M.E. Feldman, E.R. Zunder, D.D. Goldenberg, O. Williams, R. Loewith, D. Stokoe, A. Balla, B. Toth, T. Balla, W.A. Weiss, R.L. Williams, K.M. Shokat, A pharmacological map of the PI3-K family defines a role for p110alpha in insulin signaling, Cell 125 (2006) 733-747. 
[15] C.M. Lynch, L.A. Leandry, R.W. Matheny, Jr., Lysophosphatidic acid-stimulated phosphorylation of PKD2 is mediated by PI3K p110beta and PKCdelta in myoblasts, $\mathrm{J}$ Recept Signal Transduct Res 33 (2013) 41-48.

[16] C. Murga, S. Fukuhara, J.S. Gutkind, A novel role for phosphatidylinositol 3-kinase beta in signaling from G protein-coupled receptors to Akt, J Biol Chem 275 (2000) 1206912073.

[17] M. Hayakawa, H. Kaizawa, H. Moritomo, T. Koizumi, T. Ohishi, M. Okada, M. Ohta, S. Tsukamoto, P. Parker, P. Workman, M. Waterfield, Synthesis and biological evaluation of 4-morpholino-2-phenylquinazolines and related derivatives as novel PI3 kinase p110alpha inhibitors, Bioorg Med Chem 14 (2006) 6847-6858.

[18] K.G. Huwiler, T. Machleidt, L. Chase, B. Hanson, M.B. Robers, Characterization of serotonin 5-hydroxytryptamine-1A receptor activation using a phospho-extracellularsignal regulated kinase 2 sensor, Anal Biochem 393 (2009) 95-104.

[19] T.A. Kost, J.P. Condreay, R.S. Ames, S. Rees, M.A. Romanos, Implementation of BacMam virus gene delivery technology in a drug discovery setting, Drug Discov Today 12 (2007) 396-403.

[20] R. Ames, J. Fornwald, P. Nuthulaganti, J. Trill, J. Foley, P. Buckley, T. Kost, Z. Wu, M. Romanos, BacMam recombinant baculoviruses in $\mathrm{G}$ protein-coupled receptor drug discovery, Receptors Channels 10 (2004) 99-107.

[21] R.W. Matheny, Jr., M.L. Adamo, Effects of PI3K catalytic subunit and Akt isoform deficiency on mTOR and p70S6K activation in myoblasts, Biochem Biophys Res Commun 390 (2009) 252-257. 
[22] M.M. Bradford, A rapid and sensitive method for the quantitation of microgram quantities of protein utilizing the principle of protein-dye binding, Anal Biochem 72 (1976) 248254.

[23] R.W. Matheny, Jr., M.A. Riddle-Kottke, L.A. Leandry, C.M. Lynch, M.N. Abdalla, A.V. Geddis, D.R. Piper, J.J. Zhao, Role of phosphoinositide 3-OH kinase p110beta in skeletal myogenesis, Mol Cell Biol 35 (2015) 1182-1196.

[24] B.D. Manning, L.C. Cantley, AKT/PKB signaling: navigating downstream, Cell 129 (2007) $1261-1274$.

[25] Y.K. Lee, O.J. Park, Regulation of mutual inhibitory activities between AMPK and Akt with quercetin in MCF-7 breast cancer cells, Oncol Rep 24 (2010) 1493-1497.

[26] S. Kovacic, C.L. Soltys, A.J. Barr, I. Shiojima, K. Walsh, J.R. Dyck, Akt activity negatively regulates phosphorylation of AMP-activated protein kinase in the heart, J Biol Chem 278 (2003) 39422-39427.

[27] H. Hirai, H. Sootome, Y. Nakatsuru, K. Miyama, S. Taguchi, K. Tsujioka, Y. Ueno, H. Hatch, P.K. Majumder, B.S. Pan, H. Kotani, MK-2206, an allosteric Akt inhibitor, enhances antitumor efficacy by standard chemotherapeutic agents or molecular targeted drugs in vitro and in vivo, Mol Cancer Ther 9 (2010) 1956-1967.

[28] S.A. Hawley, A.E. Gadalla, G.S. Olsen, D.G. Hardie, The antidiabetic drug metformin activates the AMP-activated protein kinase cascade via an adenine nucleotideindependent mechanism, Diabetes 51 (2002) 2420-2425.

[29] B.J. Krawiec, G.J. Nystrom, R.A. Frost, L.S. Jefferson, C.H. Lang, AMP-activated protein kinase agonists increase mRNA content of the muscle-specific ubiquitin ligases MAFbx and MuRF1 in C2C12 cells, Am J Physiol Endocrinol Metab 292 (2007) E1555-1567. 
[30] R.L. Hurley, L.K. Barre, S.D. Wood, K.A. Anderson, B.E. Kemp, A.R. Means, L.A. Witters, Regulation of AMP-activated protein kinase by multisite phosphorylation in response to agents that elevate cellular cAMP, J Biol Chem 281 (2006) 36662-36672.

[31] Y. Dagon, E. Hur, B. Zheng, K. Wellenstein, L.C. Cantley, B.B. Kahn, p70S6 kinase phosphorylates AMPK on serine 491 to mediate leptin's effect on food intake, Cell Metab 16 (2012) 104-112. 


\section{Figure Legends}

Figure 1. PI3K p110a regulates AMPK phosphorylation in C2C12 myoblast. (A) Myoblasts were exposed to the indicated concentrations of p110 $\alpha$ inhibitor (p110 ai) for 4-hours. Lysates were immunoblotted with the indicated antibodies. (B) Myoblasts were treated with $5 \mathrm{nM}$ control siRNA (si-Con) or $5 \mathrm{nM}$ siRNA directed against p110 $\alpha$ (si-p110 $\alpha$ ) as described in Materials and Methods. Lysates were immunoblotted with the indicated antibodies. Blots are representative of three independent experiments. (C) Quantification of blots shown in panel B (means \pm SEMs; $n$ $=3$ independent experiments; Student's t-test; **, $P<0.01$ ). (D) Myoblasts were transduced with vector control (Vector), constitutively-active p110 $\alpha$ (p110 $\alpha$-E545K) or kinase-dead p110 $\alpha$ (p110 $\alpha-K 802 R)$ as described in Materials and Methods. Lysates were immunoblotted with the indicated antibodies. Blots are representative of three independent experiments. (E) Quantification of blots shown in panel D (means \pm SEMs; $n=3$ independent experiments; oneway ANOVA with Dunnett test post hoc; $* *, P<0.01 ; * * *, P<0.001)$.

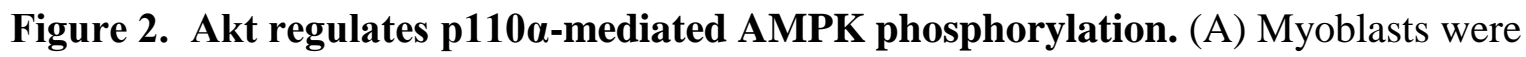
exposed to the indicated concentrations of MK2206 for 4-hours. Lysates were immunoblotted with the indicated antibodies. (B) Myoblasts transduced with vector control or p110 $\alpha-E 545 \mathrm{~K}$ were treated with MK2206 or DMSO for 4-hours prior to harvest for protein lysates. Blots are representative of three independent experiments. (C) Quantification of blots shown in panel B (means \pm SEMs; $\mathrm{n}=3$ independent experiments; one-way ANOVA with Dunnett test post hoc; $* * *, P<0.001)$. 
Figure 3. Effects of oligomycin and serum starvation on p110a-deficient myoblasts. (A) Control or p110 $\alpha$-deficient myoblasts were treated with $500 \mathrm{nM}$ oligomycin for 30 -minutes and then harvested for protein lysates. Blots are representative of at least four independent experiments. (B) Quantification of blots shown in panel A (means \pm SEMs; $n=4-6$ independent experiments; one-way ANOVA with Dunnett test post hoc; ${ }^{*}, P<0.05$; **, $P<$ 0.01 ; *** $P<0.001$ ). (C) Control or p110 $\alpha$-deficient myoblasts were exposed to serum-free (SF) media for the indicated times and then harvested for protein lysates. Blots are representative of three independent experiments. (D) Quantification of blots shown in panel C (means \pm SEMs; $\mathrm{n}=3$ independent experiments; two-way ANOVA with Bonferroni test post hoc; *, $P<0.05 ; * *, P<0.01)$.

Figure 4. Model of p110 $\alpha$-mediated AMPK phosphorylation. p110 $\alpha$ negatively regulates AMPK T172 phosphorylation independent of Akt through a yet-unidentified mechanism. p110 $\alpha$ also regulates AMPK S485/491 phosphorylation in an Akt-dependent manner. AMPK phosphorylation at S485/491 was associated with decreased AMPK T172 phosphorylation under conditions of $\mathrm{p} 110 \alpha$ hyper-activation, but this association was not observed in $\mathrm{p} 110 \alpha$ deficient myoblasts, suggesting that AMPK S485/491-mediated inhibition of T172 does not occur under all conditions (dashed line). 
A

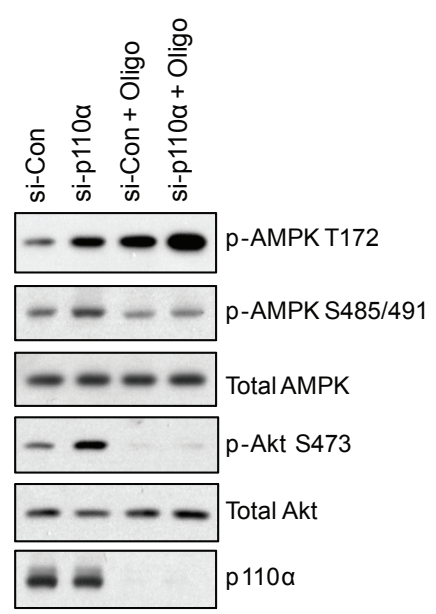

B
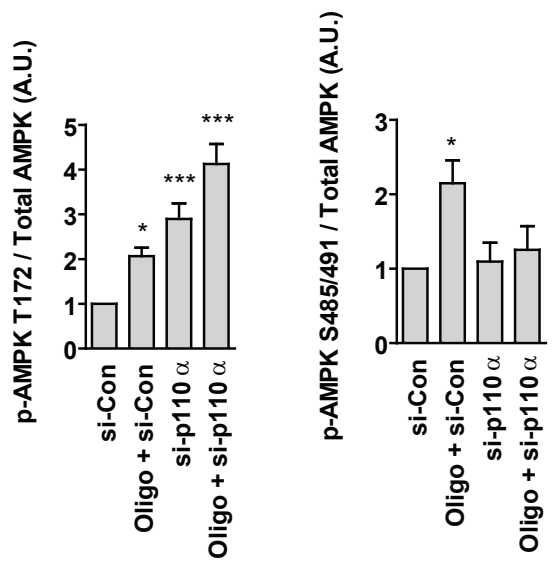

D
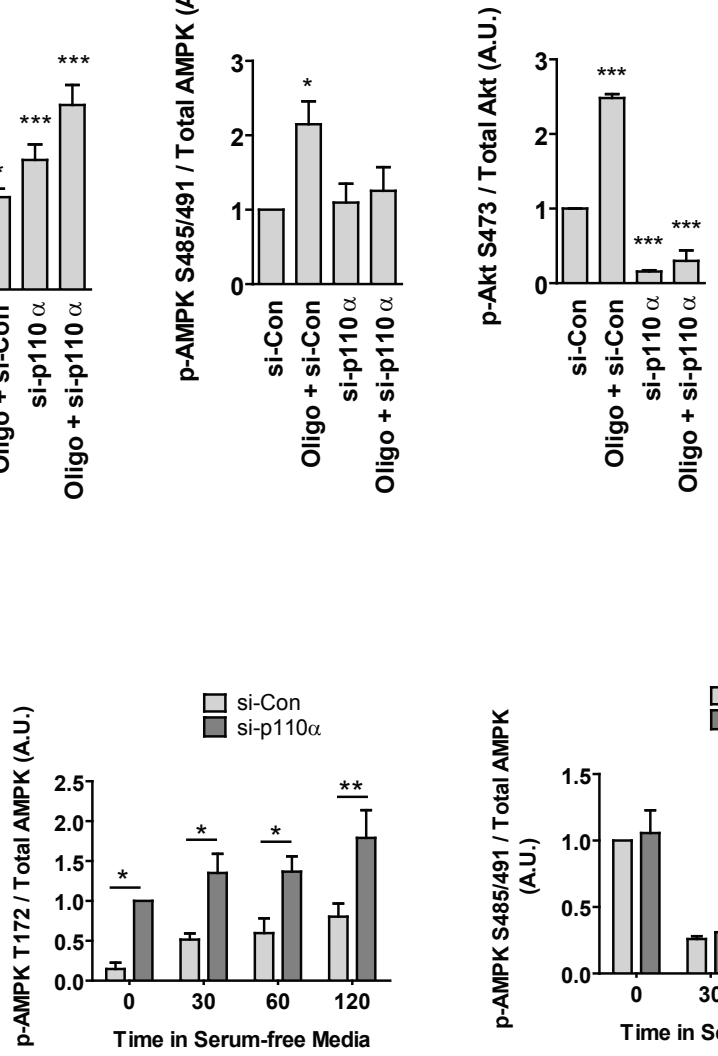

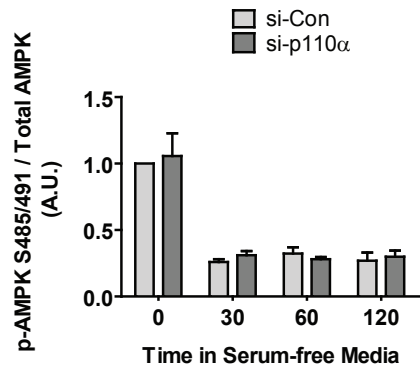

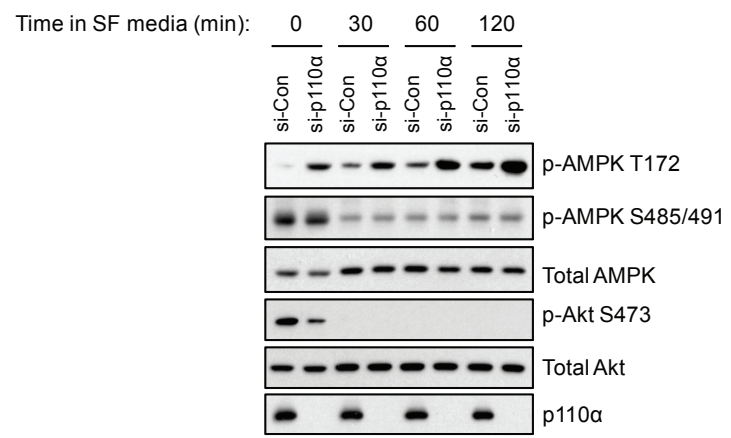


Firgigker 4

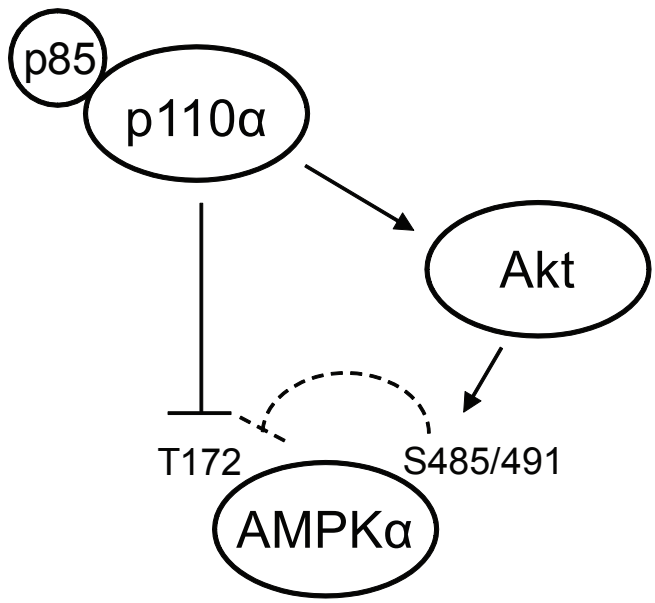




\section{Conflict of Interest - Matheny}

Click here to download Conflict of Interest: Matheny_coi_disclosure.pdf 
*Conflict of Interest - Abdalla
Click here to download Conflict of Interest: Abdalla_coi_disclosure.pdf

*Conflict of Interest - Abdalla
Click here to download Conflict of Interest: Abdalla_coi_disclosure.pdf

Click here to download Conflict of Interest: Abdalla_coi_disclosurepdf 
*Conflict of Interest - Geddis
Click here to download Conflict of Interest: Geddis_coi_disclosure.pdf

*Conflict of Interest - Geddis
Click here to download Conflict of Interest: Geddis_coi_disclosure.pdf

Click here to download Conflict of Interest: Geddis_coi_disclosurepdf

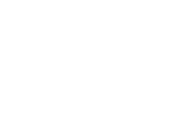

$\sqrt{2}$ (1) (1) (1) (1) (1) (1) (1) .

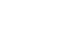

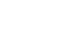

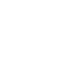

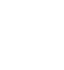

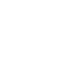

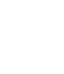

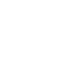

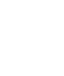

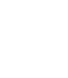

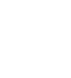

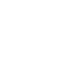

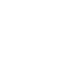

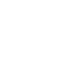

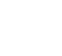


erest: Leandry coi disc

\begin{abstract}
Click here to download Conflict of Interest: Leandry_coi_disclosure.pdf
\end{abstract}

